\title{
Correction to: Brain Connect 2017;7(6):382-389, DOI:10.1089/brain.2016.0480
}

N THE July/August 2017 ISSUE of Brain Connectivity (vol. 7, no. 6, pp. 382-389, DOI:10.1089/brain.2016.0480), the title of the article currently entitled "Value of Frequency Domain Resting-State Functional Magnetic Resonance Imaging Metrics Amplitude of Low-Frequency Fluctuation and Functional Amplitude of Low-Frequency Fluctuation in the Assessment of Brain Tumor-Induced Neurovascular Uncoupling,', by Shruti Agarwal, et al., contains a typographical inaccuracy. The title should read:

"Value of Frequency Domain Resting-State Functional Magnetic Resonance Imaging Metrics Amplitude of Low-Frequency Fluctuation and Fractional Amplitude of Low-Frequency Fluctuation in the Assessment of Brain Tumor-Induced Neurovascular Uncoupling,',

The online version of the article has been corrected to reflect this change.

The authors and publisher wish to apologize for this typographical error. 